\title{
Detection of Blade Mistuning in a Low Pressure Turbine Rotor Resulting from Manufacturing Tolerances and Differences in Blade Mounting
}

\author{
Florian Schönleitner, Lukas Traussnig, Andreas Marn and Franz Heitmeir \\ Institute for Thermal Turbomachinery and Machine Dynamics, Graz University of Technology, Graz 8010, Austria
}

Received: March 18, 2015 / Accepted: April 07, 2015 / Published: May 25, 2015.

\begin{abstract}
For a serious prediction of vibration characteristics of any structure, a detailed knowledge of the modal characteristic is essential. This is especially important for bladed turbine rotors. Mistuning of the blading of a turbine rotor can appear due to manufacturing tolerances or because of the blading process itself due to unequal mounting of the blades into the disk. This paper investigates the mistuning of the individual blades of a low pressure turbine with respect to the effects mentioned above. Two different rotors with different aerodynamic design of the blades were investigated. The blades were mounted to the disk with a so-called hammer head root which is especially prone to mounting irregularities. For detailed investigations, the rotor was excited with a shaker system to detect the forced response behavior of the individual blades. The measurements were done with a laser vibrometer system. As the excitation of rotor structure was held constant during measurement, it was possible to detect the line of nodes and mode shapes as well. It could be shown that the assembly process has an influence on the mistuning. The data were analyzed and compared with numerical results. For this, different contact models and boundary conditions were used. The above described characterization of modal behavior of the rotor is the basis for the upcoming aeroelastic investigations and especially for the blade vibration measurements of the rotor, turning with design and off-design speeds.
\end{abstract}

Key words: Mistuning, modal characteristics, low pressure turbine, rotor blading, numerical and experimental modal analysis.

\section{Nomenclature}

$\begin{array}{ll}E F & \text { Eigenfrequency } \\ B L & \text { Blade } \\ B L-H & \text { Blade model including bore hole } \\ E X P & \text { Experimental } \\ B & \text { Bonded contact } \\ F & \text { Frictional contact } \\ N S & \text { No separation contact } \\ F L & \text { Frictionless contact } \\ P P & \text { Pure penalty method } \\ M P C & \text { Multi point constraint } \\ N L & \text { Normal lagrange formulation } \\ A L & \text { Augmented lagrange formulation }\end{array}$

\section{Introduction}

The determination of the amplification factor due to

Corresponding author: Florian Schönleitner, M.Sc., research fields: aeroelastics, experimental and numerical investigations. E-mail: florian.schoenleitner@tugraz.at. mistuning is an important task for the safe design of turbomachinery [1]. Rotor disks of turbomachines are designed either to have an intentional mistuning, which is achieved e.g., by small blade modifications or without mistuning. However, if a rotor is designed with identical blades, actually there are small imperfections due to the manufacturing process and tolerances, material inhomogeneity and in service wear. This small imperfections cause mistuning and can increase the forced response vibration amplitudes with respect to that of the ideal case with identical blades. The risk of high cycle fatigue is then increased, therefore mistuning is subject of several experimental and numerical investigations. Ewins [2, 3], and Castanier and Pierre [4] investigated blade vibrations of bladed disks numerically. Additionally, Castanier and Pierre [4] gave an overview of the entire mistuning literature. 
Also Chan [5] summarized the status of mistuning research.

Khemiri, et al. [6] investigated the effect of small mistuning numerically using their asymptotic mistuning model that was successfully used by Martel, et al. [7] and Martel and Corral [8].

Because mistuned simulations are extremely costly and time consuming, many authors have tried to reduce the size of the computations by means of simplified descriptions of the problem. For example, simple mass-spring systems with just a few degrees of freedom have been frequently used as can be seen in Refs. [9-12]. The fundamental mistuning model has been introduced in recent years by Feiner and Griffin [13-15]. Kaza and Kielb [16-18] have been the first who investigated the effect of intentional mistuning on flutter. Heinz, et al. [19] investigated experimentally and analytically a low pressure model turbine with intentionally mistuning. Heinz, et al. [20] figured out the influence of different mistuning configurations on the circumferential blade amplitude distribution. Wagner [21] has shown that the coupling through the rotor is a very important factor with regard to the blade amplitude distribution in a blade row.

Random mistuning due to manufacturing tolerances is normally treated by means of Monte-Carlo simulations [22] or sensitivity analysis [23, 24]. Larin [25] suggested an approach for the analysis of forced vibrations for randomly mistuned bladed disks that is based on stochastic reduced basis method and hypothesis of locally distributed mistuning.

In the paper of Hemberger, et al. [26], an overview of the work regarding real mistuned bladed structures can be found. Pohle, et al. [27] compared numerical and experimental results of a mistuned system and tried to verify the numerical results. In this work, the mistuning pattern due to manufacturing tolerances will be shown. Two different low pressure turbine rotor disks with hammer head blade root design are experimentally investigated and the results are compared with numerical results considering different contact models.

\section{Experimental Setup}

Two different low pressure turbine rotors (rotor \#1 and rotor \#2) of the test rig at Graz University of Technology, which have identical overall blade numbers but different geometries are under investigation within this paper. The blades were inserted during assembling process into the channel of the rotor disk the and finally fixed with a steel key. Fig. 1 shows schematically the design of the hammer head root blading. Basically, there are a several number of methods for the experimental modal characterization of a structure. Rotor \#1 will be characterized by the impact hammer method with roving hammer and compared to the results of shaker tests. The impact hammer characterization shows advantages in relation to a very fast and easy application. As the shaker test is beneficial for detailed investigation especially regarding mistuning in this case, rotor \#2 will be characterized with a shaker.

Kielb and Abhari [28] analyzed the blade vibratory response with and without aerodynamic loading. They summarized in their conclusion an influence of the aerodynamic damping as part of the total damping. As it is furthermore strongly dependent on the blade root geometries, it is not possible to state an influence

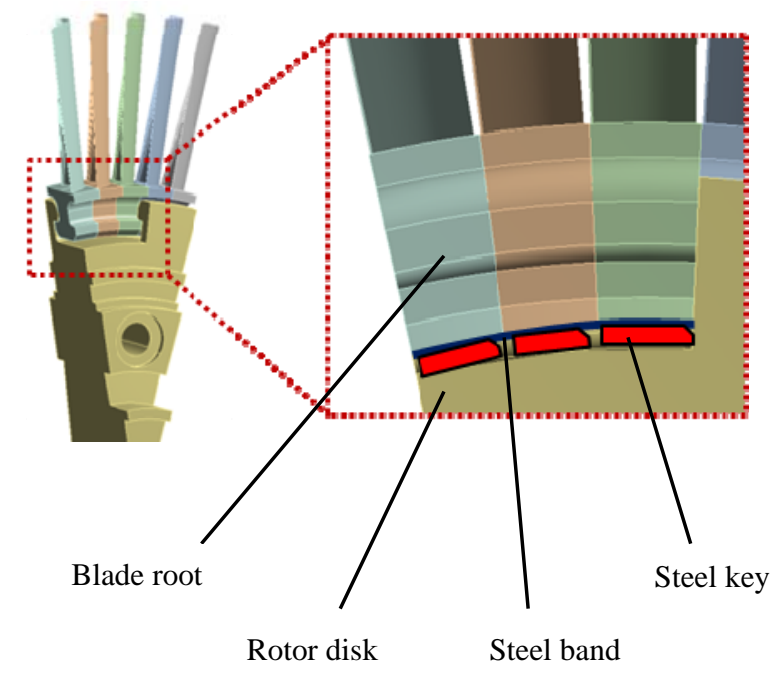

Fig. 1 Rotor assembly-blade fixation. 
of aerodynamic damping based to their investigations related to our case, as we are not able to perform vacuum tests up to now.

\section{Impact Hammer}

The low pressure turbine rotor \#1 was bedded on a soft pad to suppress influence of the bedding layer which shall be equal to a free support. Fig. 2 shows the test setup where a laser vibrometer of type Polytec OFV 303, was placed for the vibration measurement of the blading (structure response). For the modal characterization, the rotor was excited via the impact hammer of type PCB 086C03, roving hammer method on different positions on the blades as well as on the disk.

The blade vibrations were measured on each blade tip and the measurement executed in five iterations as the quality of results is strongly dependent on the hammer impact. This is due to the fact that the hammer impact is manual and hence not repeatable ideal at each measurement.

\section{Shaker}

For the shaker tests, rotors \#1 and \#2 were suspended on a steel frame and coupled with a shaker system shown in Fig. 3. The justification of rotor and shaker is important for the hanging to avoid additional tilting. This would also result in a wrong excitation of the structure.

The blade vibration measurements as a result of the excitation were carried out with the same laser

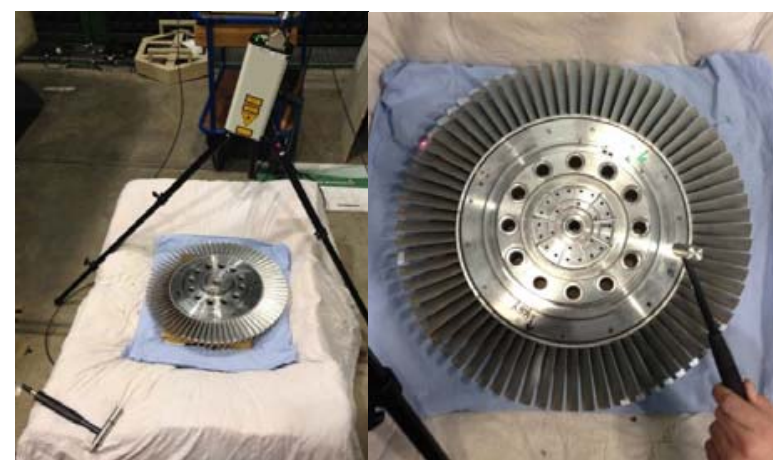

(a)

(b)

Fig. 2 (a): impact hammer PCB 086C03; (b): test setup with laser vibrometer Polytec OFV 303, excitation with roving hammer.

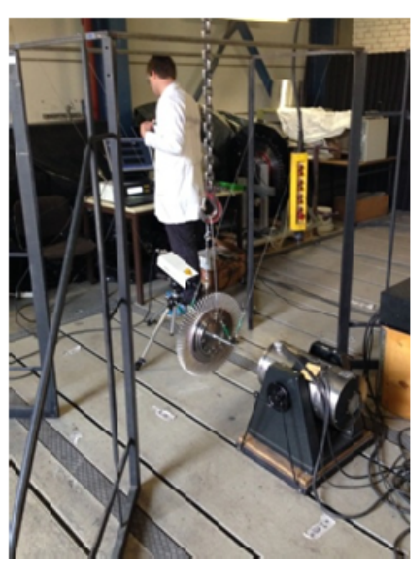

(a)

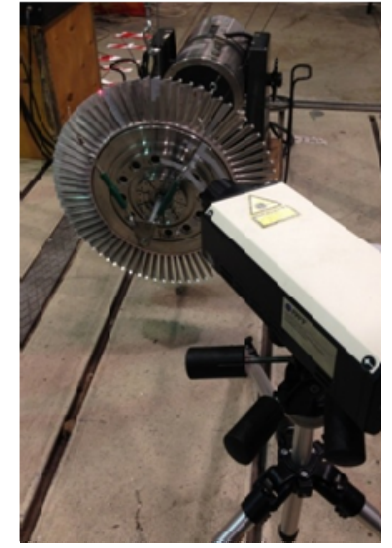

(b)
Fig. 3 Shaker tests: (a) overall test arrangement; and (b) vibration measurement.

vibrometer already used for the impact hammer tests, on different positions on the rotor blading and rotor disk as illustrated in Fig. 4. Basically, the input signal is provided by a signal generator which is amplified.

As input signal white noise was chosen. In general, it is characterized with a constant spectral density within the frequency range. So a constant excitation of the structure (input signal) can be provided over the whole test period and hence it is reproducible at any time. This is a main advantage of the shaker tests in comparison to the impact hammer method.

\section{Experimental Test Results-Impact Hammer}

The basis for analyzing the experimental data is the transfer function which was calculated by the results of the FFT (fast Fourier transform)-analysis of input and

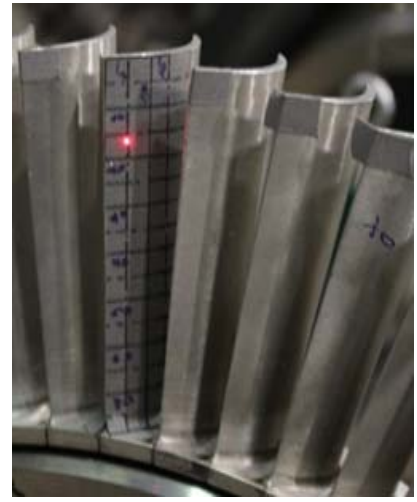

(a)

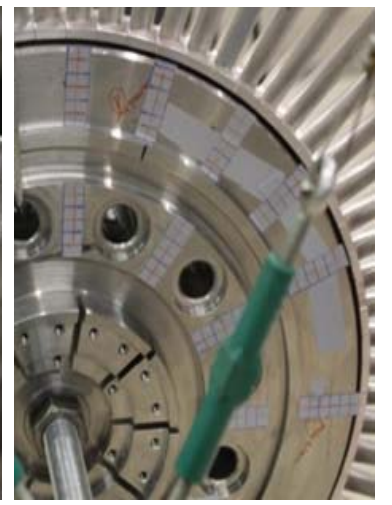

(b)
Fig. 4 Different measurement positions (a) on the blade as well as (b) on the disk. 

Manufacturing Tolerances and DIfferences in Blade Mounting

output signal. Input signal is the hammer impact for the impact hammer tests and the white noise signal for the shaker tests. The system response was measured as surface accelerations by the laser vibrometer and is therefore the output signal. Basically, the system characterization can be carried out effective and demonstrative with the complex function of type $e^{i \omega t}$.

Any input signal $U(t)$ can be fragmented via FFT in a sum of complex exponential functions which are representing the components of frequency. The signal transformation from time to frequency domain is simplified illustrated in Fig. 5.

FFT analysis shows advantages in respect to the number of analyzing steps in relation to a DFT ((discrete Fourier transformation)); calculation Fourier coefficient $N$ of order $N^{2}$ ). FFT's carry out the calculation of Fourier coefficients $N$ with a reduced number of steps $\left(N \cdot \log _{2} N\right)$. This is already well described and presented, e.g., by Shin and Hammon [29].
To avoid random noise, the results of the input signal FFT and output signal FFT were averaged. Therefore, the data are separated in identical segments. These segments are then subject of an individual FFT-analysis. Finally, all data were averaged across all transformations as illustrated in Fig. 6.

\subsection{Blade Eigenfrequencies-Rotor \#1}

The results of all rotor blades can be seen in the waterfall diagram in Fig. 7a. The same results can be also transferred in a polar diagram shown in Fig. 7b.

The first four eigenfrequencies are clearly visible and the related modes shapes are shown in Fig. 13. In general, there is a high fluctuation range of results which is also characteristic for the impact hammer method. Due to not reproducible impacts of the hammer, a deviation of results occurs.

An averaged value can be found for sure but furthermore no analysis in respect to mistuning of the

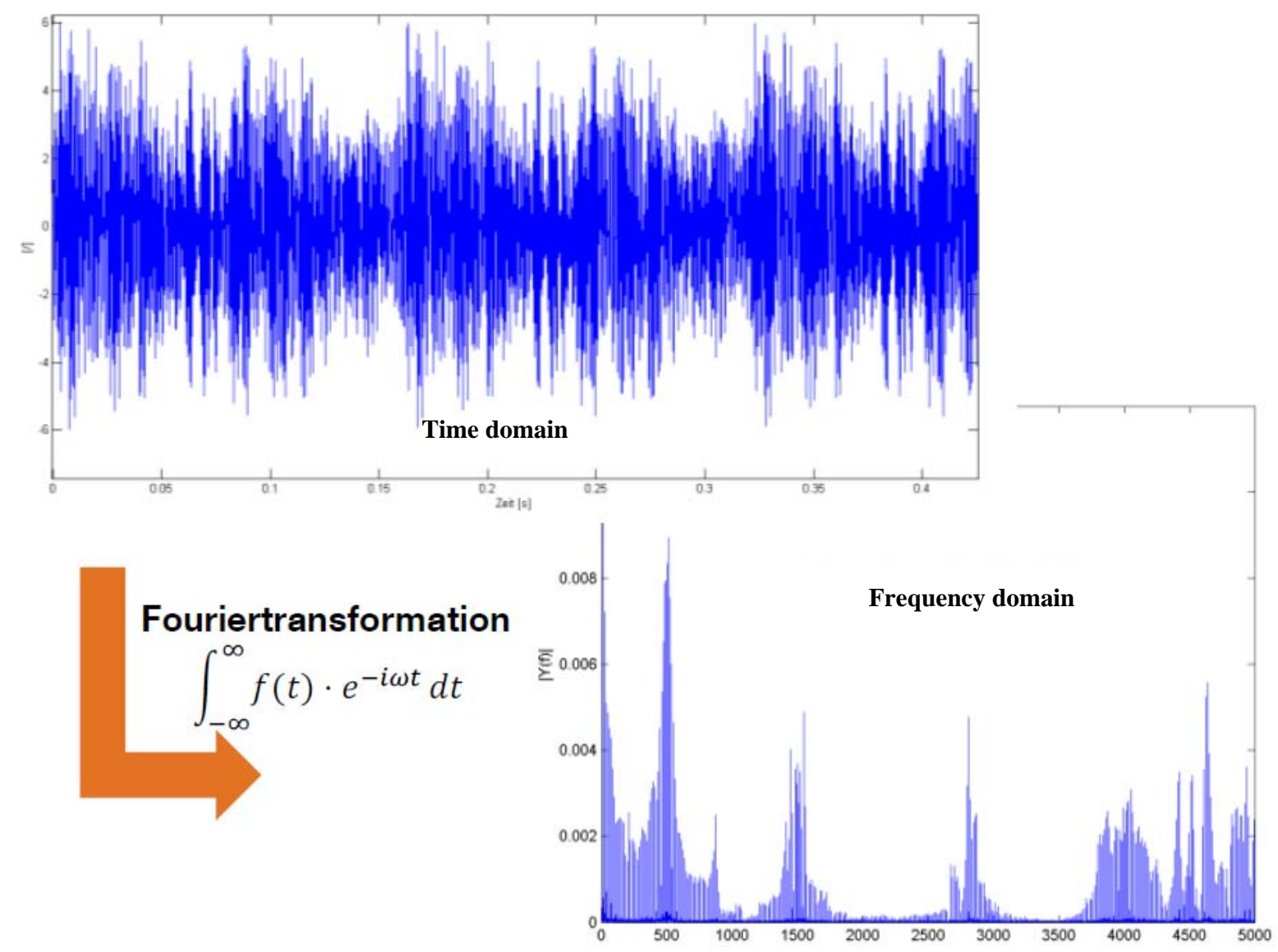

Fig. 5 Signal transformation from time to frequency domain. 


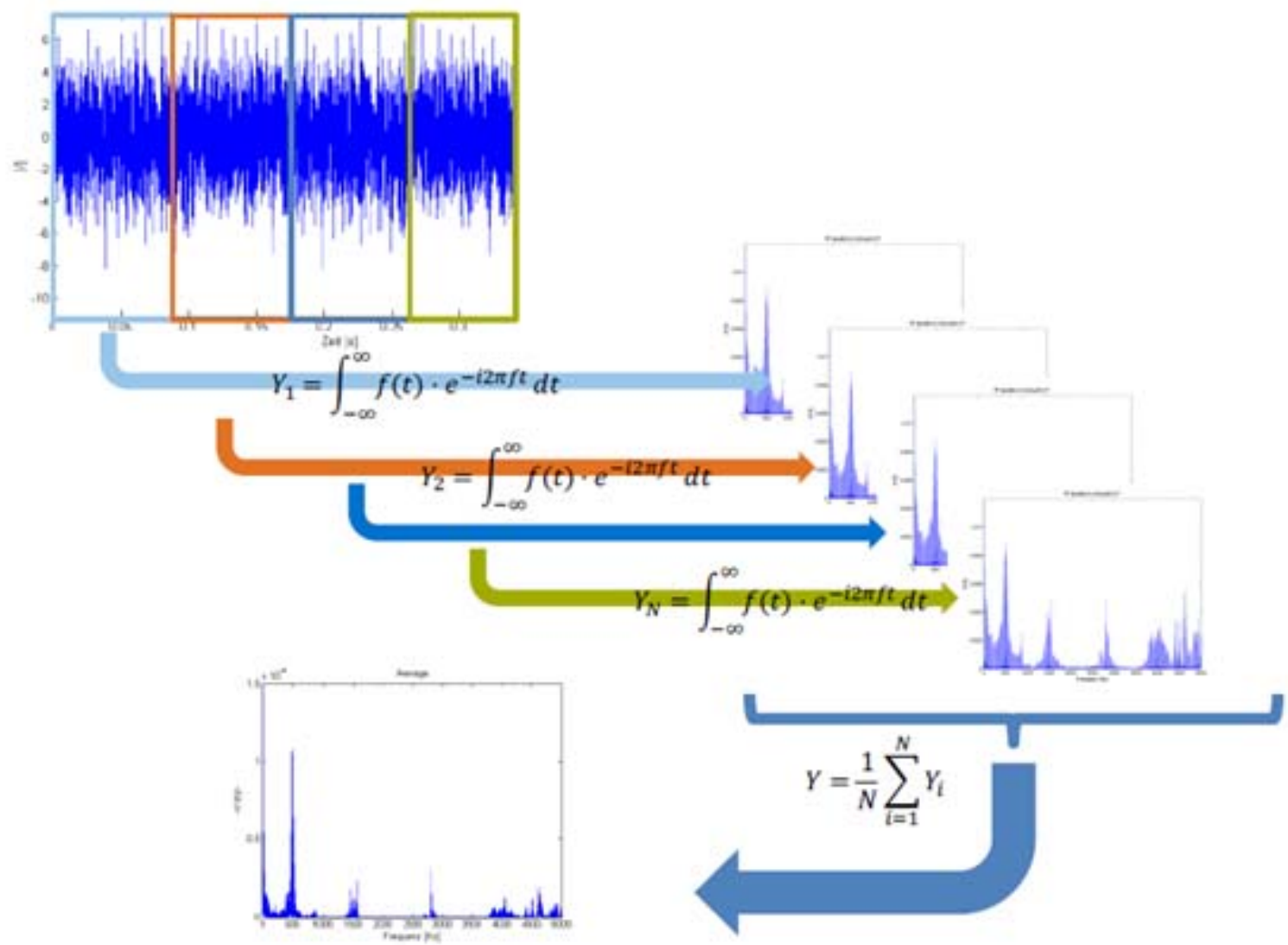

Fig. 6 Signal averaging across a number of Fourier transformations.

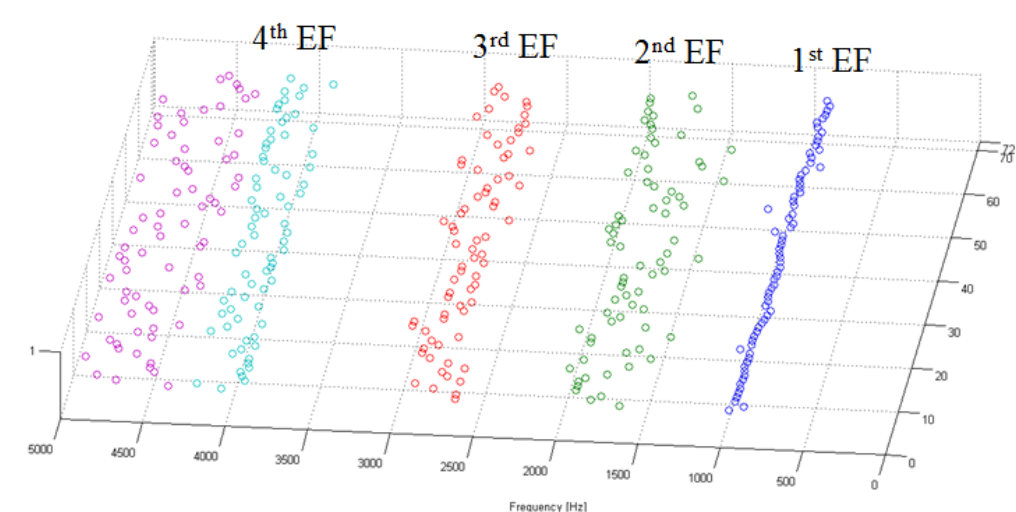

(a)

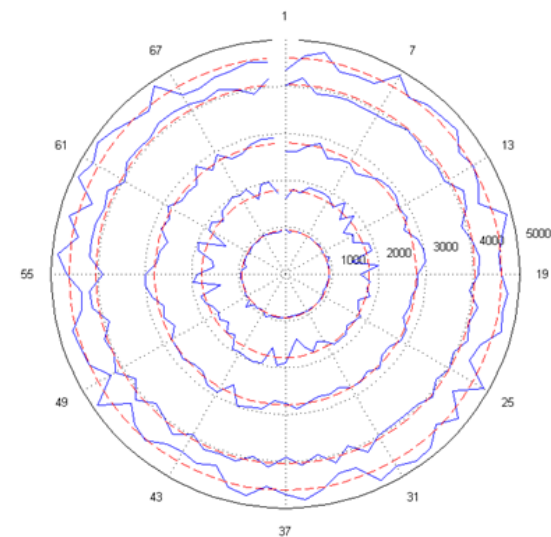

(b)

Fig. 7 (a) waterfall diagram; and (b) polar diagram of rotor \#1.

low pressure turbine rotor is possible. It also can be stated that the highest fluctuation range can be noticed at the 2nd eigenfrequency. This can be also clearly seen in the polar diagram.

In the following chapters, it will be shown that the mistuning effect especially influences the 2nd mode, so a tendency can already be noticed with the impact hammer method.

It can be summarized that the impact hammer method for the experimental modal characterization of structures is a very fast method for estimating the modal characteristics. It is characterized by a very easy test setup with the problem of the repeatability which causes high fluctuation ranges of results. 


\section{Experimental Test Results—Shaker}

Test results of the shaker were analyzed as already described in Section 3. Rotor \#1 as well as rotor \#2 was under investigation at the shaker tests. Due to a constant input excitation signal, the shaker test is predestined for investigations regarding mistuning. All analyses within this paper are focused on the blade eigenfrequencies. Rotor disk-blade coupling effects were not investigated.

\subsection{Blade Eigenfrequencies-Rotor \#1}

The results of all rotor blades can be seen in the waterfall diagram in Fig. 8a. The same results can be also transferred in a polar diagram shown in Fig. 8b where a mistuning of the rotor due to manufacturing tolerances and the blade mounting become visible. Fig. 8 shows the first four eigenfrequencies, clearly noticeable in comparison to the results of the impact hammer, is a smaller fluctuation range of results due to the constant input signal.

By trend again, the 2nd mode shows the highest deviations related to the averaged eigenfrequency out of the result of all blades.

The polar diagram visualizes this effect as well and also a mistuning especially of the right half of the rotor becomes visible in all modes.

\subsection{Blade Eigenfrequencies-Rotor \#2}

Basically, both rotor blade geometries are quite similar. Therefore, the results of averaged eigenfrequencies show similar results when comparing Figs. 8 and 9.

Nevertheless again, the first four eigenfrequencies become clearly visible where the fluctuation range decreases in comparison to rotor \#1. Again, the 2nd mode shows the highest fluctuation ranges.

When analyzing waterfall and polar diagram according to Fig. 9, the mistuning of rotor \#2 can be identified as well.

In comparison to rotor \#1, rotor \#2 shows a moderate mistuning. If the tolerances are in the same range, the rotor was manufactured by same supplier; the rotor assembly was improved. As the blade roots are fixed with a key, the pre stressing of the blading in fact is not identical.

\section{Comparison of the Experimental Results}

Fig. 10 shows a comparison between the modal characterization of low pressure turbine rotor \#1 with impact hammer method in Fig. 10a and the shaker test in Fig. 10b. Average values of the eigenfrequencies show similar results. Conspicuous is the high fluctuation range of the impact hammer due to an uneven excitation process.

Both shows the highest fluctuation ranges of mode 2 where the influence of mistuning patterns is high in comparison to all other modes.

The mistuning effect becomes clearly visible when

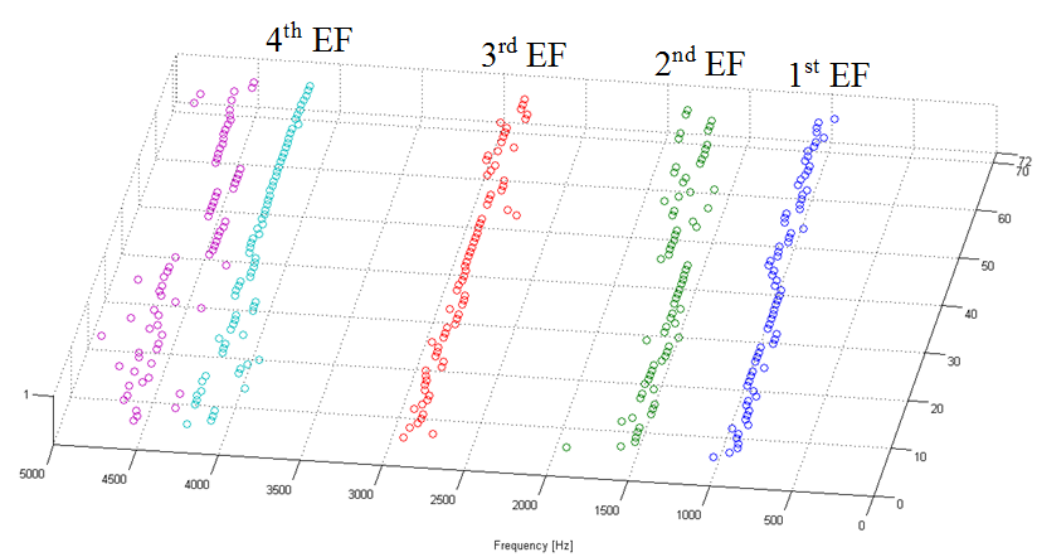

(a)

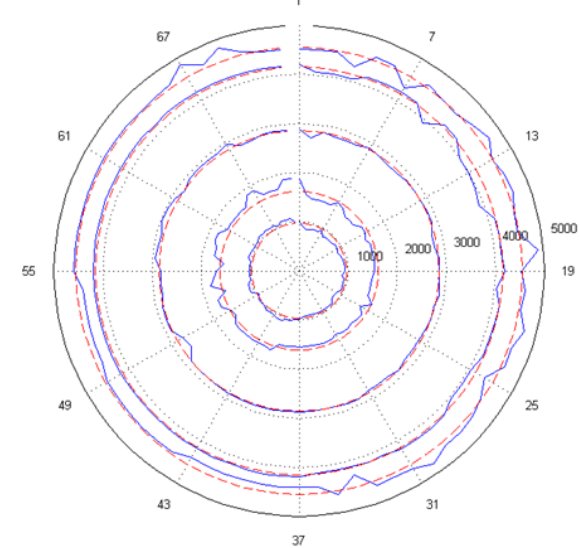

(b)

Fig. 8 (a) waterfall diagram; and (b) polar diagram of rotor \#1. 

Manufacturing Tolerances and DIfferences in Blade Mounting

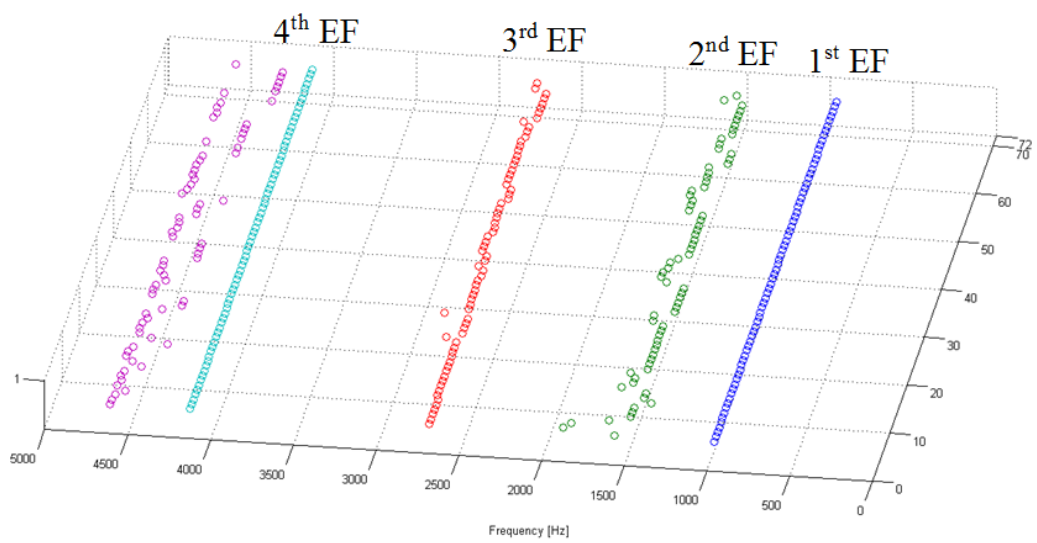

(a)

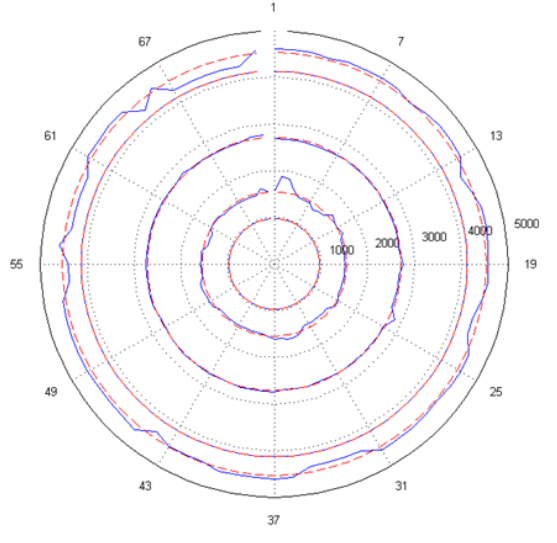

(b)

Fig. 9 (a) waterfall diagram; and (b) polar diagram of rotor \#2.

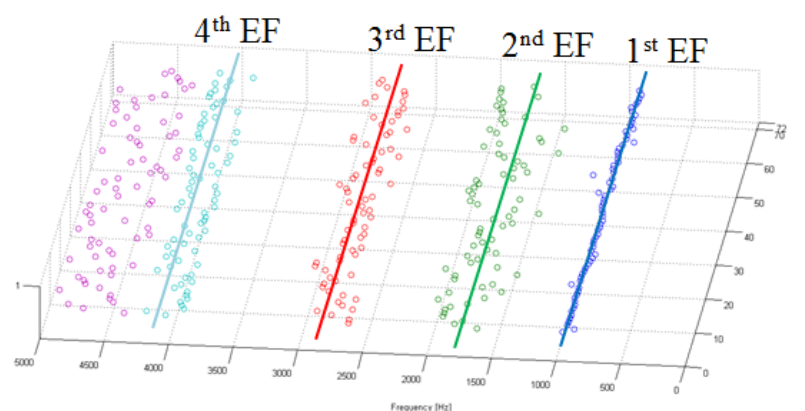

(a)

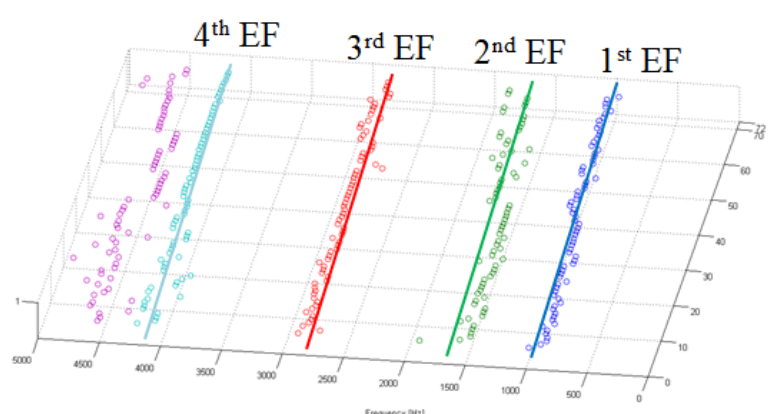

(b)

Fig. 10 Experimental results: (a) impact hammer; and (b) shaker test of rotor \#1.

illustrating the results in a polar plot as shown in Fig. 10 again for rotor \#1.

As already seen in the Fig. 9, mode 2 shows the highest dependency.

Due to the geometry of a hammer head root, potential mistuning can be occurred as a result of manufacturing tolerances. Especially the undefined gap of the lateral guidance between rotor disk and blade root, which does exist in fact, results in a mistuning seen in the 2nd EF.

Besides manufacturing tolerances, the blade mounting can cause undefined pre-stressing of the blade root and is another reason for mistuning and is an important factor of the rotor disk-blade coupling. This is furthermore a challenge in the numerical modelling. On one hand, the blade is fixed by a key, inserting manually and clamping the roots in radial direction.

On the other hand, the pre-stressing between the blade roots in circumferential direction is an additional result of the clamping process. These effects can only be minimized by an elaborateness assembling process. According to Fig. 11, rotor \#1 shows a higher mounting quality on the left half.

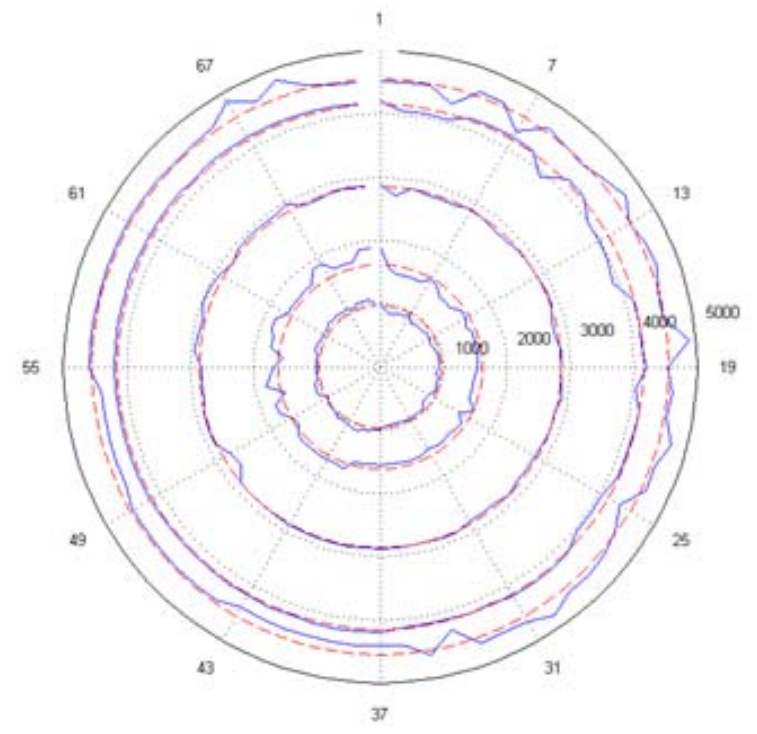

Fig. 11 Polar diagram, Rotor \#1. 
Basically, the material pairing as 3rd influence of mistuning can be stated. The investigated low pressure turbine rotors \#1 and \#2 were designed with a rotor disk out of steel and an aluminium blading. The damping of modes furthermore is strongly dependent on this fact.

All in all, it is very difficult to separate this several number of influence factors. Neither the manufacturing process nor the assembling process can be performed perfectly.

As concluded above, the 2nd eigenfrequency is mostly influenced by mistuning effects. When setting up the numerical model boundary conditions and contact formulations are essential for a serious prediction in general.

The following chapter shows the strong dependency of the 2nd eigenfrequency in relation to the boundary conditions of the blade root.

\section{Numerical Investigations}

The numerical analyses were carried out with commercial code of ANSYS 13.0. Different models of the low pressure turbine rotor \#2 as illustrated in Fig. 12 were considered. Starting with a fully assembled turbine rotor model (72BL) also a 1 blade model (1BL), a five blade model (5BL) and different 3 blade models (3BL and 3BL-H) considering varying rigidities of the rotor disk were computed. All models are hexagonal dominated meshed with quad elements. All numerical investigations are at a rotational speed of $0 \mathrm{rpm}$ to be able to compare them with the experimental results.

The lack of centrifugal forces which would result in an increase of the tightness of the contact forces is not under investigation within this paper as the experimental results were carried out at latent conditions as well.

On selected faces as seen in Fig. 13, different contact models and formulation types were applied. For a serious prediction, a face splitting of contact regions as well as a contact sizing are important. The contacts between the blade roots and the lateral guidance of blade root and disk are of particular interest. Mistuning effects are strongly dependent on these contacts and furthermore the eigenfrequencies of related modes are influenced and show the highest deviation in comparison to the experimental results as described in Section 6.2.

\subsection{Mode Shapes of the Rotor Blades}

Fig. 14 shows the mode shapes of the first four eigenfrequencies of the rotor blading of rotors \#1 and \#2.

In general, the knowledge of the system characteristics of any structure is important for further detailed investigations. Basically, the modal characteristics are on one hand an important parameter during the design

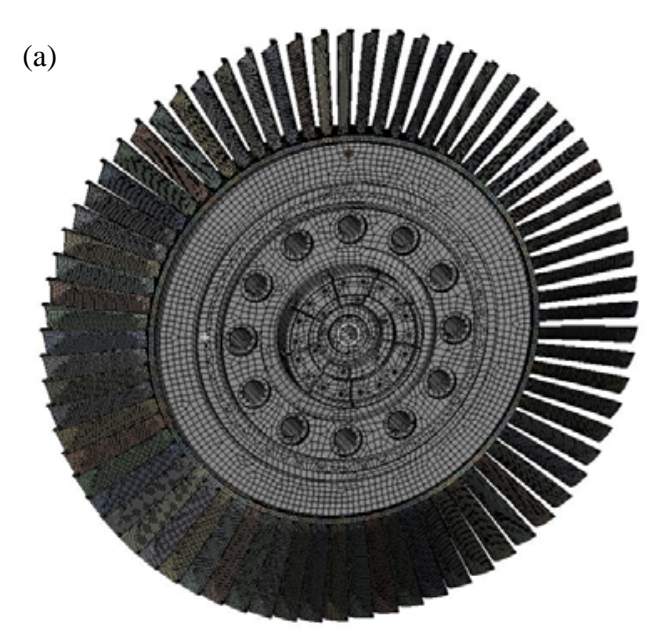

(b)

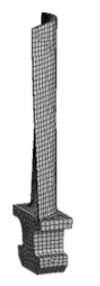

(d)

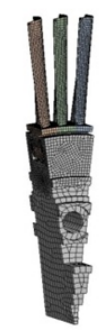

(c)

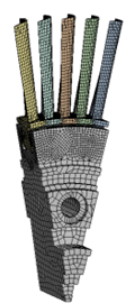

(e)

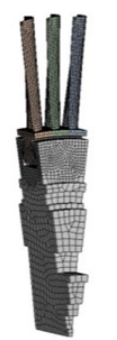

Fig. 12 Different FE (finite element)-models of the low pressure turbine rotor \#2. 


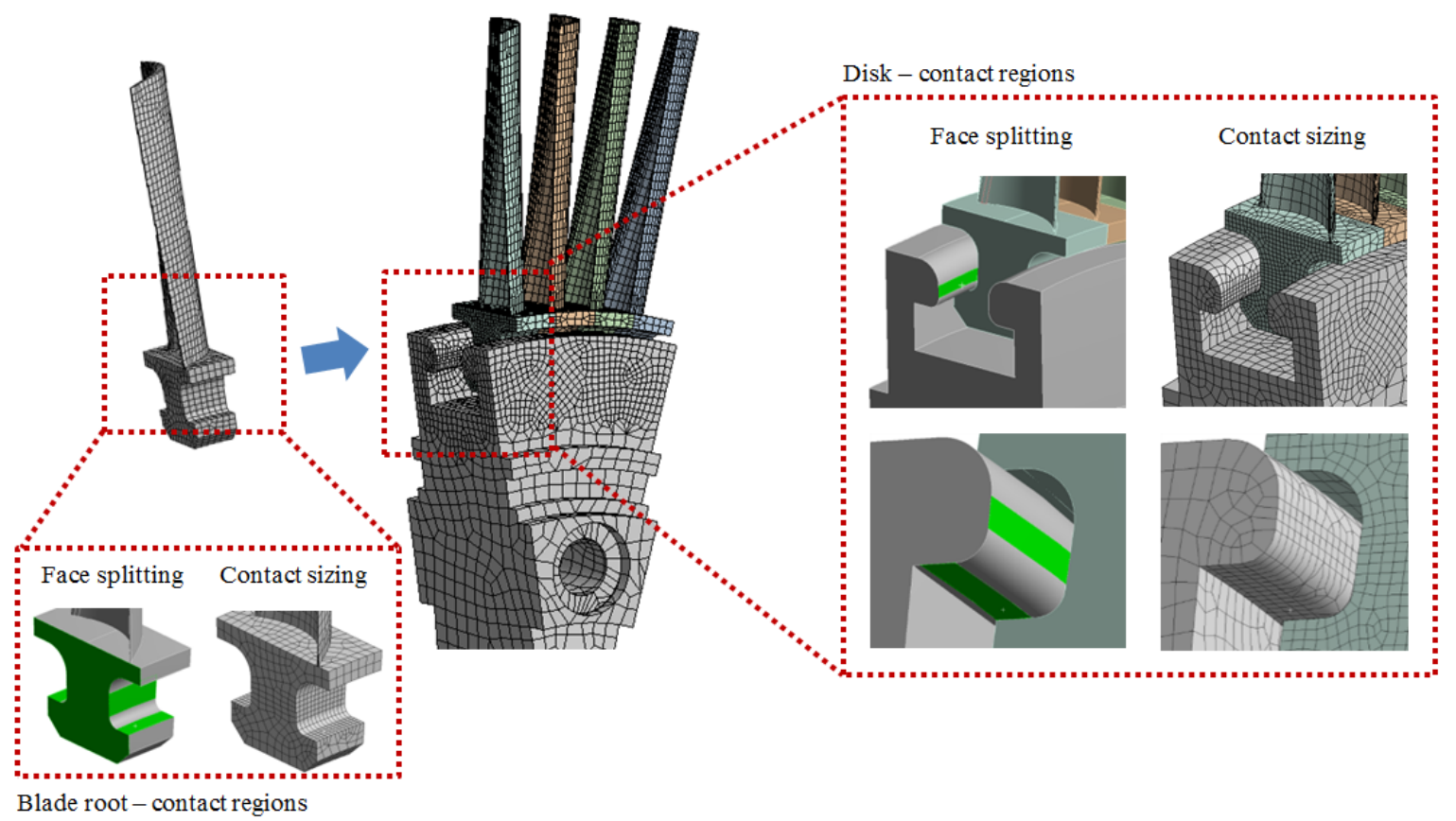

Fig. 13 Contact regions and boundary conditions of the FE-model.

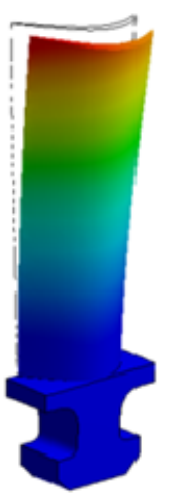

1st EF

$967 \mathrm{~Hz}$

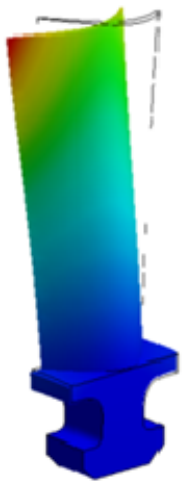

2nd EF

$1548 \mathrm{~Hz}$

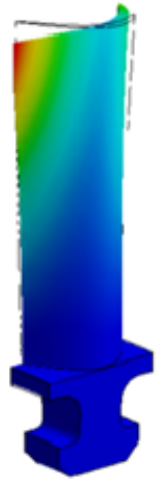

3rd EF

$2716 \mathrm{~Hz}$

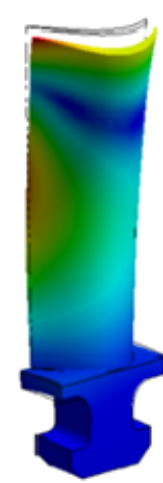

4th EF

$4131 \mathrm{~Hz}$

Fig. 14 Blade mode shapes; numerical plot (with experimental test result of rotor \#2).

process especially of rotating machines. On the other hand, the behavior under operation conditions is of special interest to guarantee safe operations. Projected on blade mechanics as presented within this paper the mode shapes plays an essential role for experimental blade vibration measurements. For these investigations, an optimum placement of strain gauges is required for a high quality of results. Therefore, the whole setup is based on the modal blade characteristics.

The first eigenfrequency is the first bending mode in circumferential direction. The second show the first bending mode in flow direction followed by the first torsional mode. The fourth eigenfrequency show the second bending mode in circumferential direction. As all other modes are at higher frequency ranges, the result discussion will be focused on the illustrated modes.

\subsection{Contact Formulation Study}

As ANSYS is providing different contact types as 

Manufacturing Tolerances and DIfferences in Blade Mounting

well as mathematical formulations, a several number of combinations of these boundary conditions are suitable. All contact types are linearized and therefore non-linear effects were not considered. The main aim was to find a good agreement of numerical results in comparison to the experimental evaluated eigenfrequencies with very simplified numerical models. Table 1 shows the results of the relative deviation of the numerical results in comparison to the averaged experimental values of eigenfrequencies for the first four shape modes and different simplified models as introduced before.

The results of the 2nd eigenfrequency are not satisfying and would need further investigations. Chosen simplification, especially for the contact of the lateral guidance between rotor disk and blade root is not suitable for a serious prediction of this mode.

The fact, that manufacturing tolerances and the blade mounting in respect to different blade root pre-stressing cannot be considered with these models make things worse.

As these are basically the reasons of a mistuning of the rotor in reality, it is furthermore a challenge in modelling the correct boundary conditions with FEM. Further investigations are necessary and could start with a more realistic contact type in future, such as a non-linear frictional contact followed by considering manufacturing tolerances.

Fig. 15 shows the comparison of results representative for the 2nd eigenfrequency. All numerical results show higher values by trend and moreover too high deviations. The 1 blade model (1BL) is very simplified. Depending on the disk rigidity, the 3 blade models show similar deviations also in comparison to the 5 blade model (5BL) which is recommended for further investigations such as FSI (fluid structure interaction) analysis where simplified models are required when computational resources are limited. This is also the case for the fully assembled rotor model in this study, where simplified contact formulations result in higher deviation of results in relation to the experimental and the other numerical results as well.

If there would be a rating of all investigated simplified models and their results in comparison to the experimental data, it can be summarized that the 1 blade model shows very good preliminary results with a fast setup including the weakness of maybe too simplified boundary conditions. The 3 blade model for this turbine rotor geometry is problematic, because of modeling correct disk rigidities. For further

Table 1 Relative deviation between experimental data and numerical results.

\begin{tabular}{|c|c|c|c|c|c|c|c|c|c|c|c|c|c|c|c|}
\hline EXP & 1BL & \multicolumn{4}{|c|}{ 3BL-H } & \multicolumn{4}{|c|}{$3 \mathrm{BL}$} & \multicolumn{4}{|c|}{$5 \mathrm{BL}$} & \multicolumn{2}{|c|}{$72 \mathrm{BL}$} \\
\hline EXP & $\mathrm{B}$ & B-MPC & N S.PP & FL-AL & F-AL & B-MPC & NS-PP & FL-AL & F-AL & B-MPC & NS.PP & FL-AL & F-AL & B-MPC & NS_PP \\
\hline 967 & $-9,4 \%$ & $-2,8 \%$ & $-3,5 \%$ & $-3,6 \%$ & $-3,0 \%$ & $-2,8 \%$ & $-4,2 \%$ & $-4,5 \%$ & $-3,2 \%$ & $-2,8 \%$ & $-4,2 \%$ & $-4,2 \%$ & $-2,9 \%$ & $1,4 \%$ & $-10,7 \%$ \\
\hline 1548 & $12,3 \%$ & $22,1 \%$ & $18,2 \%$ & $17,3 \%$ & $19,9 \%$ & $23,3 \%$ & $18,9 \%$ & $17,7 \%$ & $20,6 \%$ & $22,9 \%$ & $18,4 \%$ & $18,1 \%$ & $21,7 \%$ & $24,9 \%$ & $21,1 \%$ \\
\hline 2716 & $4,0 \%$ & $5,6 \%$ & $4,4 \%$ & $4,3 \%$ & $5,1 \%$ & $6,5 \%$ & $5,1 \%$ & $4,7 \%$ & $5,7 \%$ & $6,2 \%$ & $4,8 \%$ & $4,8 \%$ & $5,9 \%$ & $-0,6 \%$ & $-2,9 \%$ \\
\hline 4131 & $1,3 \%$ & $-3,7 \%$ & $-4,4 \%$ & $-4,5 \%$ & $-4,0 \%$ & $5,4 \%$ & $3,8 \%$ & $3,4 \%$ & $5,0 \%$ & $0,7 \%$ & $-0,7 \%$ & $-0,7 \%$ & $0,5 \%$ & $2,0 \%$ & $-5,5 \%$ \\
\hline
\end{tabular}

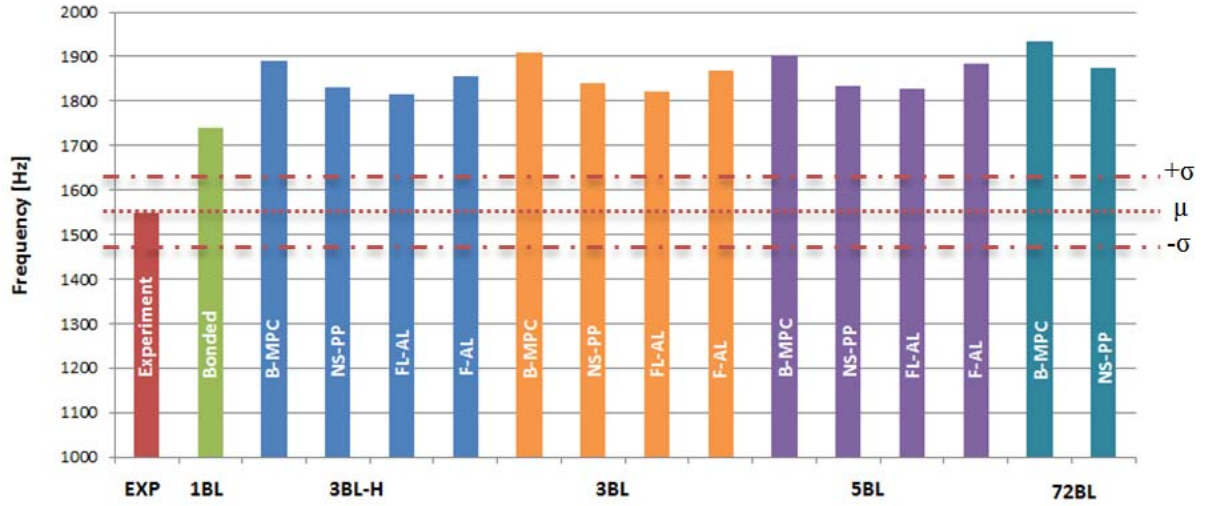

Fig. 15 Results for the 2nd eigenfrequency-numerical vs. experimental results.

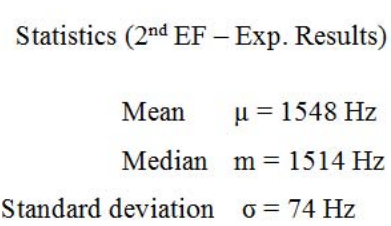

Standard deviation $\sigma=74 \mathrm{~Hz}$ 
investigations such as FSI analysis the 5 blade model is recommend which represents correct model characteristics after all simplifications.

\section{Conclusions}

This paper presented different experimental and numerical methods for the modal characterization of structures carried out on a low pressure turbine rotor. Impact hammer tests show good preliminary results with a very fast test arrangement and execution. Shaker tests are especially required for detailed analysis regarding mistuning.

In general, mistuning is an important parameter already in earlier design phases.

Mistuning is strongly influenced by manufacturing tolerances and the blade mounting. Manufacturing tolerances which are sufficient for the structural design may be able to cause unfavorable aerodynamic conditions. To prevent flutter issues, an optimal aerodynamic design in combination with structural demands is required. Additionally, the blade mounting which is strongly depended on the blade root design affects the mistuning of rotor blading. A hammer head root in combination with steel keys inserted manually, which was presented in this paper, shows a weakness of undefined positions after fastening.

Numerical analyses are based on perfect tuned structural configurations, so an identical behavior of all blades is the results.

Basically, mistuning breaks the balance of all aero elastic parameters on one hand which may be able to have advantages regarding flutter characteristics on the other hand. Therefore, the mistuning technology represents an opportunity to avoid flutter instability without impacting on aero-performance and weight.

All in all, mistuning can be detected in modal analyses of structures easily and results were presented in this paper for a low pressure turbine rotor.

Especially the second eigenfrequency is strongly influenced due to various mistuning effects. This can be also seen in the numerical analysis, where simplified contact modelling of the relevant contact region shows no satisfying results. Furthermore, simplified numerical models with different contact types as well as with mathematical formulations were presented. All in all, these numerical models show a good agreement with the experimental data.

\section{Acknowledgments}

This publication arises within a national funded project AdMoNt (contract No. 8390008) regarding aeroelastic investigations of low pressure turbine bladings at Graz Universtity of Technology at the Institute for Thermal Turbomachinery and Machine Dynamics. The authors therefore want to thank the FFG for supporting this project. Furthermore, the authors want to thank Prof. Dr. techn. H. P. Pirker for his great support during all experimental investigations as well as Prof. Dr. techn. Jakob Woisetschläger.

\section{References}

[1] Schönenborn, H., Retze, U., Ziller, G., and Waniczek, P. 2010. "Experimental and Analytical Mistuning Analysis of a Blisk at Lab Conditions and under Rig Conditions Using Tip Timing." In Proceedings of the ASME TurboExpo, 855-64.

[2] Ewins, D. 2009. "The Mistuned Blade Vibration Problem Re-visited." In Proceedings of the 12th International Symposium on Unsteady Aerodynamics, Aeroacoustics and Aeroelasticity of Turbomachines, 323-4.

[3] Ewins, D. 1991. "The Effect of Blade Mistuning on Vibration Response-A Survey." Presented at the IFToMM 4th International Conference on Rotordynamics, Prague.

[4] Castanier, M., and Pierre, C. 2006. "Modeling and Analysis of Mistuned Bladed Disk Status and Emerging Directions.” Journal of Propulsion and Power 22 (2): 384-96.

[5] Chan, Y. J. 2009. "Variability of Blade Vibration in Mistuned Bladed Discs.” Ph.D. thesis, Imperial College.

[6] Khemiri, O., Martel, C., and Corral, R. 2014. "Forced Response of Mistuned Bladed Disks: Quantitative Validation of the Asymptotic Description.” Journal of Propulsion and Power 30 (2): 397-406.

[7] Martel, C., Corral, R., and Llorens, J. 2008. "Stability Increase of Aerodynamically Unstable Rotors Using Intentional Mistuning.” Journal of Turbomachinery 130 (1): 011006. 

Manufacturing Tolerances and DIfferences in Blade Mounting

[8] Martel, C., and Corral, R. 2009. “Asymptotic Discribtion of Maximum Mistuning Amplification of Bladed Disk Forced Response.” Journal of Engineering for Gas Turbines and Power 131: 022506.

[9] Sinha, A. 1997. "Computation of the Maximum Amplitude of a Mistuned Bladed Disk Assembly via Infinity Norm.” In Proceedings of the ASME International Mechanical Engineering Congress and Exposition, 427-32.

[10] Whitehead, D. 1996. "Effect of Mistuning on the Vibration of Turbomachine Blades Induced by Wakes." Journal of Mechanical Engineering Science 8 (1): 15-21.

[11] Whitehead, D. 1998. "The Maximum Factor by Which Foced Vibration of Blades Can Increase Due to Mistuning.” Journal of Turbomachinery 120 (1): 115-9.

[12] Crawley, E., and Hall, K. 1985. "Optimization and Mechanisms of Mistuning in Cascades." Journal of Engineering for Gas Turbines and Power 107 (2): 418-26.

[13] Feiner, D., and Griffin, J. 2002. “A Fundamental Model of Mistuning for a Single Family of Modes.” Journal of Turbomachinery 124 (4): 597-605.

[14] Feiner, D., and Griffin, J. 2004. "Mistuning Identification of Bladed Disks Using Fundamental Mistuning Model, Part 1: Theory.” Journal of Turbomachinery 126: 150-8.

[15] Feiner, D., and Griffin, J. 2004. "Mistuning Identification of Bladed Disks Using Fundamental Mistuning Model, Part 2: Application.” Journal of Turbomachinery 126: 159-65.

[16] Kaza, K. R., and Kielb, R. E. 1983. “Aeroelastic Characteristics of a Cascade of Mistuned Blades in Subsonic and Supersonix Flows.” Journal of Vibration, Acoustics, Stress and Reliability of Design 105: 425-33.

[17] Kaza, K. R. V., and Kielb, R. E. 1983. "Effects of Structural Coupling on Mistuned Cascade Flutter and Response.” J. Eng. Gas Turbines Power 106 (1): 17-24.

[18] Kaza, K. R. V., and Kielb, R. E. 1984. "Flutter of Turbofan Rotors with Mistuned Blades.” AIAA Journal 22 (11): 1618-25.

[19] Heinz, C., Schatz, M., Stüer, H., and Casey, M. 2010. "Experimental and Analytical Investigation of a Low Pressure Model Turbine During Forced Response
Excitation.” In Proceedings of ASME TurboExpo, 97-106.

[20] Heinz, C., Schatz, M., Stüer, H., and Casey, M. 2011. "Impact of Mistuning on the Vibration Behaviour of the Last Stage in a Model Three Stage Low Pressure Steam Turbine.” In Proceedings of ASME TurboExpo, 1047-56.

[21] Wagner, J. T. 1967. "Coupling of Turbomachine Blade Vibration through the Rotor.” Journal of Engineering for Power 89: 502-12.

[22] Beck, J., Brown, J., Cross, C., and Slater, J. 2012. "Probabilistic Mistuning Assessment Using Nominal and Geometry Based Mistuning Methods.” In Proceedings of ASME TurboExpo,1085-97.

[23] Griffin, J., and Kenyon, J. 2003. "Forced Response of Turbine Engine Bladed Disks and Sensitivity to Harmonic Mistuning.” Journal of Turbomachninery 125: 113-20.

[24] Petrov, E. P. 2007. “A Sensitivity-Based Method for Direct Stochastic Analysis of Nonlinear Forced Response for Bladed Disks with Friction Interfaces.” Journal of Engineering for Gas Turbines and Power 130 (2): 022503.

[25] Larin, O. O. 2010. "Forced Vibrations of Bladings with the Random Technological Mistuning.” In Proceedings of ASME TurboExpo, 667-72.

[26] Hemberger, D., Filsinger, D., and Bauer, H. J. 2012. "Investigation on Maximum Amplitude Amplfication Factor of Real Mistuned Bladed Structures." In Proceedings of ASME Turbo Expo 2012: Turbine Technical Conference and Exposition, 1041-52.

[27] Pohle, L., Aschenbruck, J., Panning-von Scheidt, L., Wallaschek, J., and Seume, J. 2014. "Dynamic Behavior of a Mistuned Air Turbine: Comparison Between Simulations and Measurements.” Presented at the ASME Turbo Expo 2014: Turbine Technical Conference and Exposition, Germany.

[28] Kielb, J. J., and Abhari, R. S. 2003. "Experimental Study of Aerodynamic and Structural Damping in a Full-Scale Rotating Turbine." Journal of Engineering for Gas Turbines and Power 1025: 102-12.

[29] Shin, K., and Hammond, J. K. 2008. Fundamentals of Signal Processing for Sound and Vibration Engineers. New York: John Wiley and Sons Ltd. 